\title{
Diboson resonance and vector-like quark searches at ATLAS and CMS
}

\author{
Miaoran $\mathbf{L u}^{a, *}$ \\ ${ }^{a}$ University of Science and Technology of China, \\ No.96, JinZhai Road Baohe District,Hefei,Anhui, 230026,P.R.China \\ E-mail: miaoran.lu@cern.ch
}

This letter reports some latest results of Diboson resonance and vector-like quark searches at ATLAS and CMS. The diboson searches includes hunting for $V V, V H$, and $H H$ resonances in fully hadronic or semi-leptonic final states. A novel model-independent dijet resonance search with weak supervision is also introduced. The vector-like quark section includes searches for single VLQ production that has been carried out recently.

The Eighth Annual Conference on Large Hadron Collider Physics-LHCP2020 25-30 May, 2020

online

\footnotetext{
${ }^{*}$ Speaker
} 


\section{Introduction}

Many beyond standard model theories has been proposed predicting new heavy bosons like the spin-0 radion in Randall-Sundrum (RS) framework [1, 2], the spin- $1 W^{\prime}$ and $Z^{\prime}$ in HVT model [3], and the spin 2 Kaluza-Klein graviton $\left(G_{\mathrm{KK}}\right)$ in the bulk RS model [4-6]. These heavy bosons could decay into a pair of standard model bosons like $W, Z, H$, or $\gamma$. Thus a search for the diboson resonance is a good probe of beyond standard model physics.

The vector like quarks (VLQ) are heavy non-chiral quarks with symmetric left and right handed couplings to standard model particles. VLQs are predicted in many BSM theories and could help to stabilize the higgs mass and offer a potential solution to the hierarchy problem. The decay of the VLQs provides a rich set of possible signatures.

\section{Diboson resonance and vector-like quark searches}

A search for heavy resonances decaying into a $W$ or $Z$ boson and a Higgs boson [7] was carried out based on the sample collected by the ATLAS detector, corresponding to $139 \mathrm{fb}^{-1}$ of integrated luminosity. The search exploits jet substructure techniques to study the fully-hadronic $q q b b$ final state which results from the dominant decay modes of the $W / Z$ and Higgs bosons. No significant excess of events is observed over the expected background and upper limits on the cross section for $p p \rightarrow W^{\prime} \rightarrow W H$ and $p p \rightarrow Z^{\prime} \rightarrow Z H$ are set at $95 \% \mathrm{CL}$ as shown in Figure 1. These results translate into lower-mass limits on $W^{\prime}\left(Z^{\prime}\right)$ bosons of $2.90 \mathrm{TeV}(2.20 \mathrm{TeV})$ in the context of the weakly coupled HVT model A and of $3.20 \mathrm{TeV}(2.65 \mathrm{TeV})$ in the context of the strongly coupled HVT model B, at $95 \% \mathrm{CL}$.
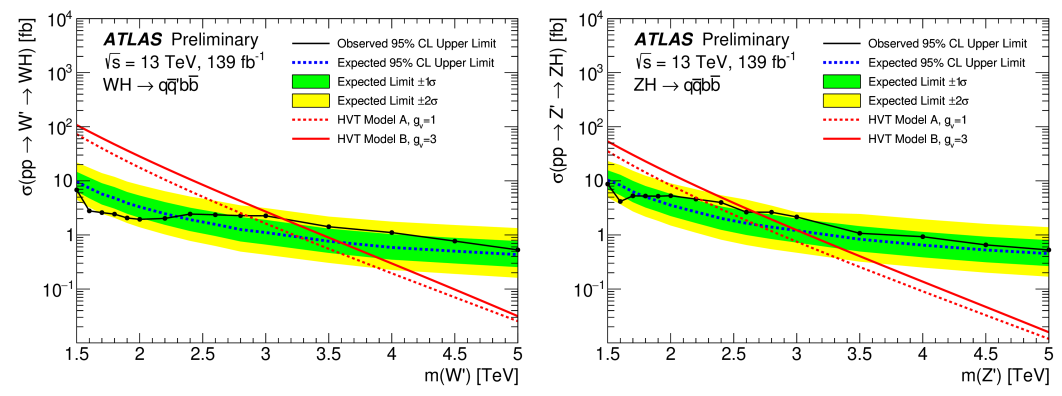

Figure 1: Observed and expected 95\% CL upper limits on the cross section for $p p \rightarrow V^{\prime} \rightarrow V H$ in the $W H$ (left) and $Z H$ (right) channels.

Searches for the production of heavy diboson resonances in the 0-lepton, 1-lepton and 2-lepton final states [8] are performed using data with an integrated luminosity of $139 \mathrm{fb}^{-1}$ collected by the ATLAS detector. The data are found to be in good agreement with background expectations. Upper limits on the production of heavy resonances in the mass range $300-5000 \mathrm{GeV}$ through gluon-gluon fusion, Drell-Yan or vector-boson fusion processes are derived for Standard Model extensions with an additional neutral scalar, a heavy vector triplet, or warped extra dimensions. Combining the $W W$ and $Z Z$ decay modes, the observed $95 \%$ confidence-level upper limit on $p p \rightarrow X \rightarrow V V$ for the $\mathrm{ggF}(\mathrm{VBF})$ process ranges from $1.8(0.60) \mathrm{pb}$ at $300 \mathrm{GeV}$ to $0.38(0.23) \mathrm{fb}$ at $5 \mathrm{TeV}$ for an RS radion 
and from $1.4(0.40) \mathrm{pb}$ at $300 \mathrm{GeV}$ to $0.26(0.30) \mathrm{fb}$ at $5 \mathrm{TeV}$ for an RS graviton. These observed limits set lower mass limits of $3.2 \mathrm{TeV}$ for the ggF production of an RS radion, and $2.0(0.76) \mathrm{TeV}$ for the $\mathrm{ggF}(\mathrm{VBF})$ production of an RS graviton. For the production of $W^{\prime}$ and $Z^{\prime}$ bosons in the HVT framework, the observed upper limit on the cross section $p p \rightarrow W^{\prime} \rightarrow W Z$ varies from 1.9 $\mathrm{pb}$ at $300 \mathrm{GeV}$ to $0.16 \mathrm{fb}$ at $5 \mathrm{TeV}$ for DY production and from $1.3 \mathrm{pb}$ at $300 \mathrm{GeV}$ to $0.35 \mathrm{fb}$ at 4 $\mathrm{TeV}$ for VBF production. Similarly, the limits on the cross section $p p \rightarrow Z^{\prime} \rightarrow W W$ are observed to vary from $0.9 \mathrm{pb}$ at $300 \mathrm{GeV}$ to $0.18 \mathrm{fb}$ at $5 \mathrm{TeV}$ for DY production and from $1.36 \mathrm{pb}$ at $300 \mathrm{GeV}$ to $0.25 \mathrm{fb}$ at $4 \mathrm{TeV}$ for VBF production. In the benchmark Model A (Model B), these cross-section upper limits exclude the ggF production of a $W^{\prime}$ boson with $m\left(W^{\prime}\right)<3.9(4.3) \mathrm{TeV}$ and a $Z^{\prime}$ boson with $m\left(Z^{\prime}\right)<3.5(3.9) \mathrm{TeV}$.

A model-independent dijet resonance search with weak supervision from ATLAS is also carried out recently [9]. This analysis uses a machine learning anomaly detection procedure that does not rely on any signal model and search for new particles in final state jets and dijet resonances at the same time. The core of this analysis is a technique called classification without labels (CWola) that uses a network trained to distinguish the difference of $M_{j j}$ shape between a given signal region and the two neighboring side-band regions. For jets produced from Lorentz-boosted heavy-particle decays, this search is more sensitive than the inclusive dijet search and extends the coverage of the all-hadronic diboson search to regions away from the SM boson masses.

A search is presented for resonances with masses above $1.2 \mathrm{TeV}$ that decay to $W W, Z Z$, or $W Z$ boson pairs from CMS collaboration [10]. Each of the two bosons decays into one large-radius jet, yielding dijet final states. No evidence is found for a signal, and upper limits on the resonance production cross section are set as shown in Figure 2.
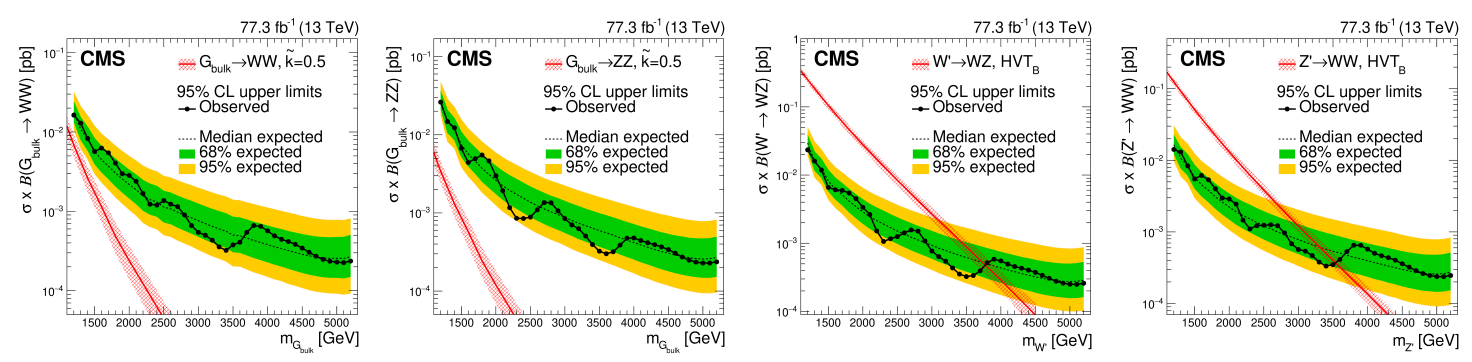

Figure 2: Observed and expected 95\% CL upper limits on the product of the production cross section and the branching fraction, obtained after combining categories of all purities with $77.3 \mathrm{fb}^{-1}$ of $13 \mathrm{TeV}$ data, for $G_{\text {bulk }} \rightarrow W W, G_{\text {bulk }} \rightarrow Z Z, W^{\prime} \rightarrow W Z$, and $Z^{\prime} \rightarrow W W$ signals.

A search for new particles decaying to a pair of Higgs bosons where one decays to a bottom quark pair and the other into two $W$ bosons that subsequently decay into a lepton, a neutrino, and a quark pair [11] has been carried out using a sample collected by the CMS detector, corresponding to an integrated luminosity of $35.9 \mathrm{fb}^{-1}$. The data are consistent with expected standard model background. The results are upper limits on the product of cross section and branching fraction for new bosons decaying to $H H$. The observed limit at $95 \%$ confidence level for a spin- 0 resonance ranges from $123 \mathrm{fb}$ at $0.8 \mathrm{TeV}$ to $8.3 \mathrm{fb}$ at $3.5 \mathrm{TeV}$, while the limit for a spin-2 resonance is $103 \mathrm{fb}$ at $0.8 \mathrm{TeV}$ and $7.8 \mathrm{fb}$ at $3.5 \mathrm{TeV}$. 
A search for the production of a single vector-like quark $Q$, where $Q$ can be either a $T$ or $Y$ quark, with the subsequent decay into $W b$ [12] has been carried out with the ATLAS experiment corresponding to an integrated luminosity of $36.1 \mathrm{fb}^{-1}$. The observed data distributions are compatible with the expected Standard Model background and no significant excess is observed. Limits at $95 \% \mathrm{CL}$ are set on the coupling parameters and cross-section times branching ratio as shown in Figure 3.
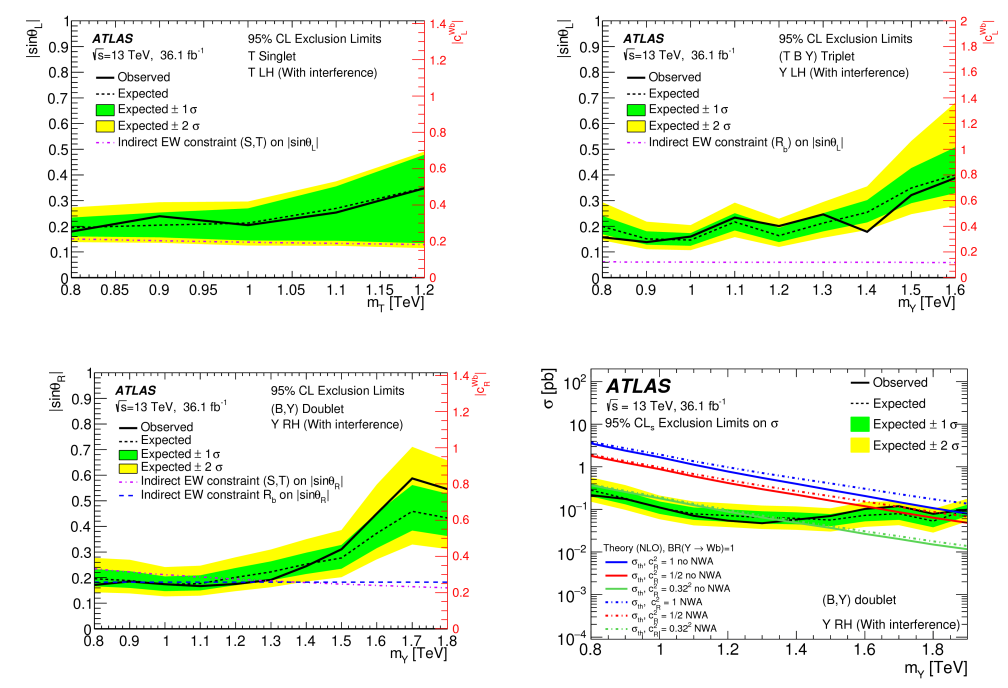

Figure 3: Observed (solid line) and expected (short-dashed line) $95 \%$ CL limits on the mixing angle $\left|\sin \theta_{\mathrm{L}}\right|$ and the coupling value $c_{\mathrm{L}}^{W b}$ for a singlet $T$-quark model assuming $\operatorname{Br}(T \rightarrow W b) \approx 0.5,\left|\sin \theta_{\mathrm{L}}\right|$ and $c_{\mathrm{L}}^{W b}$ for a $(T, B, Y)$ triplet model, $\left|\sin \theta_{\mathrm{R}}\right|$ and $c_{\mathrm{R}}^{W b}$ for a $(B, Y)$ doublet model assuming a branching ratio $\operatorname{Br}(Y \rightarrow W b)$ $=1$, and cross-section times branching ratio for the case of the right-handed $Y$ quark for a $(B, Y)$ doublet model, as a function of the VLQ mass.

A search for anomalous production of events with large $E_{\mathrm{T}}^{\text {miss }}$ and a single top-quark [13] was also carried out using data collected by the ATLAS detector in 2015 and 2016. Single production of VLQ was used as one of its signal model. No deviations with respect to SM predictions are observed and $95 \% \mathrm{CL}$ upper limits on the production cross-section of the single production of vector-like $T$ quarks decaying into $t Z(\rightarrow v \bar{v})$ is obtained. The limits are also interpreted in terms of the excluded regions in the parameter space. Couplings of the $T$ singlets to top-quarks and $W$ bosons, $c_{W}$, above 0.7 are excluded for $m_{T}=1.4 \mathrm{TeV}$ and below.

A search for a vector-like top quark partner $T$ in the electroweak single production mode with fully hadronic final states [14] has been performed using data collected by the CMS experiment in 2016 corresponding to an integrated luminosity of $35.9 \mathrm{fb}^{-1}$. No significant excess of data above the standard model background is observed and upper limits at $95 \%$ confidence level are set on $\sigma \times B r(T \rightarrow t H)$ and $\sigma \times B r(T \rightarrow t Z)$, which vary between $2 \mathrm{pb}$ and $20 \mathrm{fb}$ for $T$ masses ranging from 0.6 to $2.6 \mathrm{TeV}$ in the $T b q$ and $T t q$ production channels. The combined $T \rightarrow t H$ and $T \rightarrow t Z$ results for associated production with a bottom quark lead to constraints on $T$ quarks in the $T$ singlet model for masses below $1.00 \mathrm{TeV}$. The expected sensitivity for this model extends to $1.28 \mathrm{TeV}$ (for $30 \%$ fractional width). 


\section{References}

[1] W. D. Goldberger and M. B. Wise, Modulus Stabilization with Bulk Fields, Phys. Rev. Lett. 83 (1999) 4922, arXiv: hep-ph/9907447 [hep-ph].

[2] W. D. Goldberger and M. B. Wise, Phenomenology of a stabilized modulus, Phys. Lett. B 475 (2000) 275, arXiv: hep-ph/9911457 [hep-ph].

[3] D. Pappadopulo, A. Thamm, R. Torre and A. Wulzer, Heavy vector triplets: bridging theory and data, JHEP 09 (2014) 060, arXiv: 1402.4431 [hep-ph].

[4] L. Randall and R. Sundrum, A Large Mass Hierarchy from a Small Extra Dimension, Phys. Rev. Lett. 83 (1999) 3370, arXiv: hep-ph/9905221 [hep-ph].

[5] T. Han, J. D. Lykken and R.-J. Zhang, On Kaluza-Klein states from large extra dimensions, Phys. Rev. D 59 (1999) 105006, arXiv: hep-ph/9811350 [hep-ph].

[6] K. Agashe, H. Davoudiasl, G. Perez and A. Soni, Warped gravitons at the LHC and beyond, Phys. Rev. D 76 (2007) 036006, arXiv: hep-ph/0701186 [hep-ph].

[7] ATLAS Collaboration, Search for resonances decaying into a weak vector boson and a Higgs boson in the fully hadronic final state produced in proton-proton collisions at $\sqrt{s}=13 \mathrm{TeV}$ with the ATLAS detector, arXiv:2007.05293 [hep-ex].

[8] ATLAS Collaboration, Search for heavy diboson resonances in semileptonic final states in pp collisions at $\sqrt{s}=13 \mathrm{TeV}$ with the ATLAS detector, arXiv:2004.14636 [hep-ex].

[9] ATLAS Collaboration, Dijet resonance search with weak supervision using $\sqrt{s}=13 \mathrm{TeV} p p$ collisions in the ATLAS detector, arXiv:2005.02983 [hep-ex].

[10] CMS Collaboration, A multi-dimensional search for new heavy resonances decaying to boosted $W W, W Z$, or ZZ boson pairs in the dijet final state at $\sqrt{s}=13$ TeV, Eur. Phys. J. C 80 (2020) 237, arXiv:1906.05977 [hep-ex].

[11] CMS Collaboration, Search for resonances decaying to a pair of Higgs bosons in the HH in $b \bar{b} q \bar{q}^{\prime} \ell v$ final states at $\sqrt{s}=13 \mathrm{TeV}$, JHEP 10 (2019) 125.

[12] ATLAS Collaboration, Search for single production of vector-like quarks decaying into Wb in pp collisions at $\sqrt{s}=13 \mathrm{TeV}$ with the ATLAS detector, JHEP 05 (2019) 164, arXiv: 1812.07343 [hep-ex].

[13] ATLAS Collaboration, Search for large missing transverse momentum in association with one top-quark in pp collisions at $\sqrt{s}=13 \mathrm{TeV}$ with the ATLAS detector, JHEP 05 (2019) 041, arXiv:1812.09743 [hep-ex].

[14] CMS Collaboration, Search for electroweak production of a vector-like T quark using fully hadronic final states, JHEP 01 (2020) 036, arXiv:1909.04721 [hep-ex]. 